\title{
ON THE CONVERGENCE OF MONOTONE HURWITZ GENERATING FUNCTIONS
}

\author{
I. P. GOULDEN, MATHIEU GUAY-PAQUET, AND JONATHAN NOVAK
}

\begin{abstract}
Monotone Hurwitz numbers were introduced by the authors as a combinatorially natural desymmetrization of the Hurwitz numbers studied in enumerative algebraic geometry. Over the course of several papers, we developed the structural theory of monotone Hurwitz numbers and demonstrated that it is in many ways parallel to that of their classical counterparts. In this note, we identify an important difference between the monotone and classical worlds: fixed-genus generating functions for monotone double Hurwitz numbers are absolutely summable, whereas those for classical double Hurwitz numbers are not. This property is crucial for applications of monotone Hurwitz theory in analysis. We quantify the growth rate of monotone Hurwitz numbers in fixed genus by giving universal upper and lower bounds on the radii of convergence of their generating functions.
\end{abstract}

\section{INTRODUCTION}

Let us identify the symmetric group $\mathfrak{S}(d)$ with its right Cayley graph, as generated by the conjugacy class of transpositions. Furthermore, let us equip $\mathfrak{S}(d)$ with the edge-labelling in which each edge corresponding to the transposition $(s t)$ is marked with $t$, the larger of the two numbers interchanged. A restricted version of this labelling was introduced by Stanley [22] as an EL-labelling of the noncrossing partition lattice $\mathrm{NC}(d)$ related to parking functions; it was subsequently ported to the symmetric group by Biane [1] using a natural embedding $\mathrm{NC}(d) \rightarrow \mathfrak{S}(d)$. Figure 1 shows $\mathfrak{S}(4)$ together with the Biane-Stanley edge labelling: 2-edges are drawn in blue, 3-edges in yellow, and 4-edges in red. In general, we will refer to the edge labels of $\mathfrak{S}(d)$ as colours.

A walk on $\mathfrak{S}(d)$ is said to be monotone if the colours of the edges it traverses form a weakly increasing sequence. Once this notion has been introduced, a natural question is to count monotone walks of a given length between two given permutations. It turns out that this question, which is of a purely combinatorial nature, is closely connected to certain problems in analysis. In particular, it is known [15] that, for any $\rho, \sigma \in \mathfrak{S}(d)$ and any $N \geq d$, one has

$$
\int_{U(N)} u_{11} \ldots u_{d d} \overline{u_{1 \rho^{-1} \sigma(1)} \cdots u_{d \rho^{-1} \sigma(d)}} \mathrm{d} U=\frac{1}{N^{d}} \sum_{r=0}^{\infty}(-1)^{r} \frac{\vec{w}^{r}(\rho, \sigma)}{N^{r}},
$$

where the integration is against the Haar probability measure on the compact group of $N \times N$ complex unitary matrices $U=\left[u_{i j}\right]_{i, j=1}^{N}$, and $\vec{w}^{r}(\rho, \sigma)$ is the number of $r$-step monotone walks on $\mathfrak{S}(d)$ from $\rho$ to $\sigma$. It is a non-obvious fact that the

2010 Mathematics Subject Classification. 05A05, 05A15.

Key words and phrases. Hurwitz numbers, generating functions, walks in graphs. 


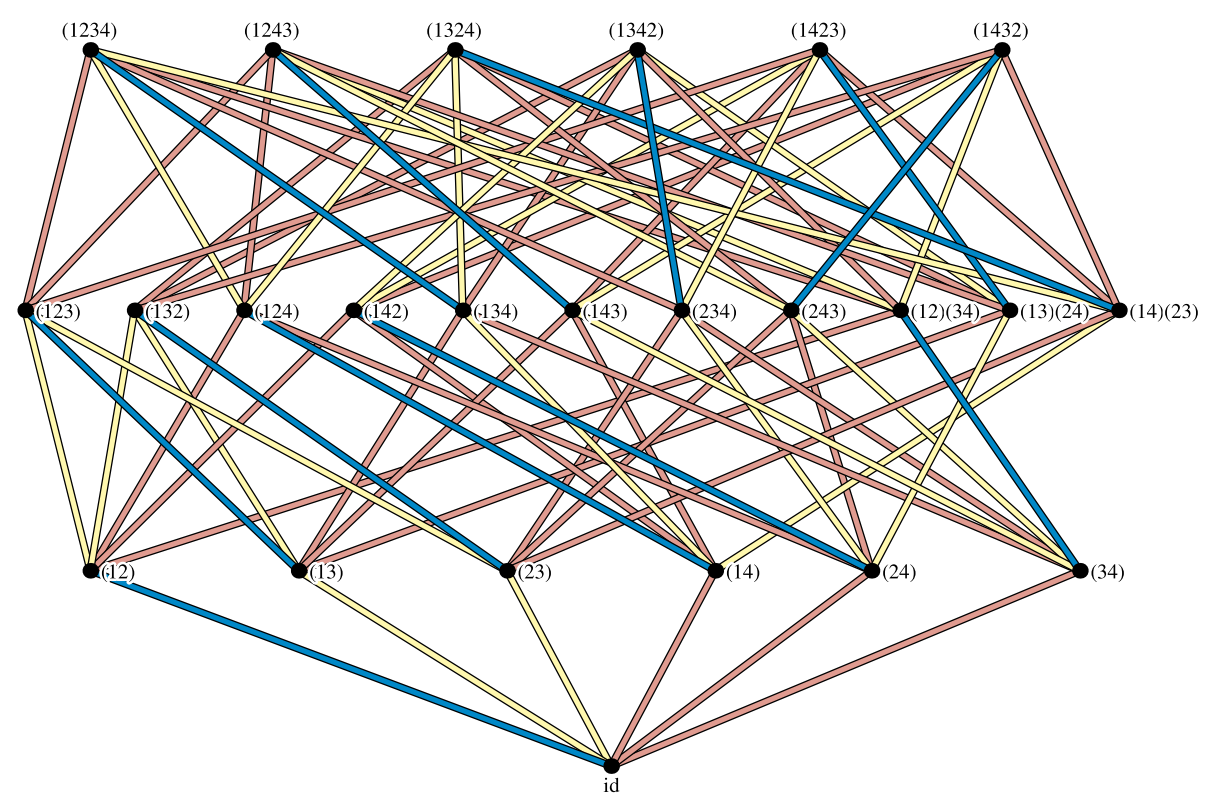

Figure 1. $\mathfrak{S}(4)$ with the Biane-Stanley edge-labelling (figure by M. LaCroix).

number $\vec{w}^{r}(\rho, \sigma)$, and hence the integral in 1.1), depends on the permutations $\rho$ and $\sigma$ only through the cycle type of $\rho^{-1} \sigma$. Moreover, as explained in [14, the formula (1.1) implies that the computation of inner products of homogeneous polynomial functions of degree $d \leq N$ in the Hilbert space $L^{2}(U(N)$, Haar) is equivalent to the enumeration of monotone walks on $\mathfrak{S}(d)$. The computation of such inner products is important in various parts of mathematical physics, including lattice quantum chromodynamics [2, 21] and string theory [11, and from this perspective (1.1) serves as a kind of Feynman diagram expansion. Polynomial integrals on $U(N)$ are also ubiquitous in random matrix theory, where in the course of various moment computations it is necessary to "integrate out" eigenvector data to get at the eigenvalue distribution of a unitarily invariant random matrix.

Another perspective on the problem of enumerating monotone walks on $\mathfrak{S}(d)$ is to consider it as a desymmetrization of the double Hurwitz problem from enumerative algebraic geometry. Given two partitions $\alpha, \beta \vdash d$, and a nonnegative integer $g \geq$ 0 , let $H_{g}(\alpha, \beta)$ denote the total number of $(2 g-2+\ell(\alpha)+\ell(\beta))$-step walks on $\mathfrak{S}(d)$, not necessarily monotone, which begin at a permutation of cycle type $\alpha$, end at a permutation of cycle type $\beta$, and have the property that their steps and endpoints together generate a transitive subgroup of $\mathfrak{S}(d)$. The numbers $H_{g}(\alpha, \beta)$ are known as the double Hurwitz numbers because, by a classical construction due to Hurwitz [12], $\frac{1}{d !} H_{g}(\alpha, \beta)$ is a weighted count of degree $d$ branched covers of the Riemann sphere $\mathbf{P}^{1}$ by a genus $g$ compact, connected Riemann surface which have ramification profile $\alpha$ over $\infty, \beta$ over 0 , and the simplest non-trivial branching over $2 g-2+\ell(\alpha)+\ell(\beta)$ additional specified points of $\mathbf{P}^{1}$, the number of which is determined by the Riemann-Hurwitz formula. It is convenient to introduce the notation 
for this number.

$$
r_{g}(\alpha, \beta)=2 g-2+\ell(\alpha)+\ell(\beta)
$$

The double Hurwitz numbers have been objects of intense study in both algebraic combinatorics and algebraic geometry, see e.g. [10, 18, 19] and references therein, and it is known that they exhibit a wide array of subtle structural properties. In [7, 8, 9], we introduced and studied the monotone double Hurwitz numbers $\vec{H}_{g}(\alpha, \beta)$, which count the same walks as the double Hurwitz numbers $H_{g}(\alpha, \beta)$, but with the monotonicity constraint imposed. In [7, 8], we demonstrated that the monotone double Hurwitz numbers enjoy a high degree of structural similarity with the classical double Hurwitz numbers, and in [9] we applied this structure to the asymptotic analysis of unitary matrix integrals via (1.1). While our analysis was purely combinatorial, geometric explanations of the concordance between monotone and classical Hurwitz theory are gradually appearing, see e.g. [4, 5].

The present note is not about structural analogies between monotone and classical Hurwitz numbers, but rather about an important quantitative difference between the two: growth rate. Obviously, the monotone Hurwitz number $\vec{H}_{g}(\alpha, \beta)$ is smaller than its classical counterpart $H_{g}(\alpha, \beta)$ - but how much smaller? While explicit formulas for Hurwitz numbers are few and far between (see the introduction of [8] for a summary of known formulas), the elementary genus zero formulas

$$
\frac{1}{d !} \vec{H}_{0}\left(1^{d}, d\right)=\frac{1}{d^{2}}\left(\begin{array}{c}
2 d-2 \\
d-1
\end{array}\right), \quad \frac{1}{d !} H_{0}\left(1^{d}, d\right)=d^{d-3},
$$

already reveal a stark distinction: the classical Hurwitz number grows superexponentially in the degree $d$, whereas its monotone analogue exhibits only exponential growth. This sharp drop in growth rate plays an important role in analytic applications of monotone Hurwitz theory [9, 16, where one needs to know that, for any fixed $g \geq 0$, the generating function

$$
\overrightarrow{\mathbf{F}}_{g}(z)=\sum_{d=1}^{\infty}\left(\sum_{\alpha, \beta \vdash d} \vec{H}_{g}(\alpha, \beta)\right) \frac{z^{d}}{d !}
$$

enumerating all genus $g$ monotone transitive walks on $\mathfrak{S}(d)$ is absolutely summable. In fact, one needs the stronger statement that the radius of convergence of $\overrightarrow{\mathbf{F}}_{g}(z)$ is bounded below by a positive universal constant, so that one may consider $\left\{\overrightarrow{\mathbf{F}}_{g}(z)\right\}$ as a family of holomorphic functions defined on a common complex domain. The goal of this note is to give a detailed proof of a precise form of this growth property.

Theorem 1. For any $g \geq 0$, the radius of convergence of the generating function $\overrightarrow{\mathbf{F}}_{g}(z)$ is at least $1 / 54$ and at most $2 / 27$.

The proof of Theorem 1 consists of two components.

The first component, which was established in [9] using the results of [7, 8], is concerns the generating function

$$
\overrightarrow{\mathbf{S}}_{g}(z)=\sum_{d=1}^{\infty} \vec{H}_{g}\left(1^{d}, 1^{d}\right) \frac{z^{d}}{d !}
$$


for genus $g$ monotone simple Hurwitz numbers $\vec{H}_{g}\left(1^{d}, 1^{d}\right)$, which count monotone transitive identity-based loops on the symmetric groups of length $r_{g}\left(1^{d}, 1^{d}\right)$. More precisely, in [9, Theorem 4.1], we proved that $\overrightarrow{\mathbf{S}}_{g}(z)$ has radius of convergence $2 / 27$ by expressing it in terms of the Gauss hypergeometric function

$$
{ }_{2} F_{1}\left(\frac{2}{3}, \frac{4}{3}, \frac{3}{2} ; \frac{27}{2} z\right) .
$$

This yields an upper bound of $2 / 27$ on the radius of convergence of $\overrightarrow{\mathbf{F}}_{g}(z)$.

The second component of the proof of Theorem 1 , which we establish in Proposition 4 of this paper, is the lower bound in Theorem 1 . This is obtained by showing that the terms of $\overrightarrow{\mathbf{F}}_{g}(z)$ are larger than those of $\overrightarrow{\mathbf{S}}_{g}(z)$ by at most an explicit exponential factor:

$$
\sum_{\alpha, \beta \vdash d} \vec{H}_{g}(\alpha, \beta) \leq 4^{d} \vec{H}_{g}\left(1^{d}, 1^{d}\right) .
$$

To prove the inequality 1.5, we make use of a sorting action of the symmetric group $\mathfrak{S}(r)$ on $r$-step walks on $\mathfrak{S}(d)$ which is in fact a close relative of Hurwitz's classical braid group action on branched coverings (see e.g. 6] for an informative discussion of braids and branched coverings).

Although the inequality 1.5 is sufficient for our purposes, it may not be sharp. In fact, we conjecture that the radius of convergence of $\overrightarrow{\mathbf{F}}_{g}(z)$ is exactly $2 / 27$ for all $g \geq 0$, so that, by Pringsheim's theorem, these generating functions have a common dominant singularity at the critical point $z_{c}=2 / 27$. This would be consistent with various similarities between Hurwitz numbers, maps on surfaces, and matrix models, where common singular behaviour of generating functions across all genera is an established phenomenon, see e.g. [18] and references therein.

Finally, we cannot resist mentioning the striking fact, pointed out to us by P. Di Francesco, that the peculiar number $2 / 27$ also occurs in the enumeration of finite groups. Namely, given a prime $p$, let $\Gamma_{p}(N)$ denote the number of isomorphism classes of groups of order $p^{N}$; it is known (see [20] and references therein) that

$$
\lim _{N \rightarrow \infty} \frac{1}{N^{3}} \log \Gamma_{p}(N)=\frac{2}{27} \log p .
$$

Any explanation of this coincidence would doubtless be very interesting.

\section{Proof of Theorem 1}

2.1. Swapping steps. Fix permutations $\rho, \sigma \in \mathfrak{S}(d)$ and a positive integer $r$, and consider the set $\operatorname{Walk}_{r}(\rho, \sigma)$ of all (not necessarily monotone or transitive) $r$ step walks from $\rho$ to $\sigma$ (assume $r$ is large enough so that this set is non-empty). Equivalently, $\operatorname{Walk}_{r}(\rho, \sigma)$ is the set of $r$-tuples

$$
W=\left(\left(s_{1} t_{1}\right), \ldots,\left(s_{r} t_{r}\right)\right), \quad s_{i}<t_{i},
$$

of transpositions such that

$$
\sigma=\rho\left(s_{1} t_{1}\right) \ldots\left(s_{r} t_{r}\right) .
$$


Given a walk $W$ as in (2.1), we refer to the list $\left(t_{1}, \ldots, t_{r}\right)$ as the spectrum of $W$, and the elements of this list as colours. Let

$$
\chi: \operatorname{Walk}_{r}(\rho, \sigma) \longrightarrow\{2, \ldots, d\}^{r}
$$

be the map which sends a walk $W$ to its spectrum $\chi(W)$.

The symmetric group $\mathfrak{S}(r)$ acts naturally on spectra $\chi \in\{2, \ldots, d\}^{r}$ by permuting colours: for each $\pi \in \mathfrak{S}(r)$ we have a corresponding operator

$$
S_{\pi}\left(t_{1}, \ldots, t_{r}\right)=\left(t_{\pi(1)}, \ldots, t_{\pi(r)}\right)
$$

on spectra, and it is clear that $S_{\pi_{1}} S_{\pi_{2}}=S_{\pi_{1} \pi_{2}}$.

One may also define an action of $\mathfrak{S}(r)$ on $\operatorname{Walk}_{r}(\rho, \sigma)$. To define this action, we begin by introducing operators $R_{i}, 1 \leq i \leq r-1$ which act on walks as follows: we set

$$
R_{i}\left(s_{1} t_{1}\right) \ldots\left(s_{i} t_{i}\right)\left(s_{i+1} t_{i+1}\right) \ldots\left(s_{r} t_{r}\right)=\left(s_{1} t_{1}\right) \ldots\left(* t_{i+1}\right)\left(* t_{i}\right) \ldots\left(s_{r} t_{r}\right),
$$

where

$$
\left(* t_{i+1}\right)=\left(s_{i} t_{i}\right)\left(s_{i+1} t_{i+1}\right)\left(s_{i} t_{i}\right) \quad \text { and } \quad\left(* t_{i}\right)=\left(s_{i} t_{i}\right)
$$

if $t_{i}<t_{i+1}$,

$$
\left(* t_{i+1}\right)=\left(s_{i+1} t_{i+1}\right) \quad \text { and } \quad\left(* t_{i}\right)=\left(s_{i+1} t_{i+1}\right)\left(s_{i} t_{i}\right)\left(s_{i+1} t_{i+1}\right)
$$

if $t_{i}>t_{i+1}$, and finally

$$
\left(* t_{i+1}\right)=\left(s_{i+1} t_{i+1}\right) \quad \text { and } \quad\left(* t_{i}\right)=\left(s_{i} t_{i}\right)
$$

if $t_{i}=t_{i+1}$. In words, the effect of $R_{i}$ is to swap the $i$ and $(i+1)$ st steps of $W$ and then conjugate the step with the higher colour by the step with the lower colour; if the two steps are the same colour, $R_{i}$ does nothing.

Proposition 2. The operators $R_{i}$ satisfy the Coxeter relations:

$$
\begin{aligned}
R_{i}^{2} & =I, \quad 1 \leq i \leq r-1 \\
R_{i} R_{i+1} R_{i} & =R_{i+1} R_{i} R_{i+1}, \quad 1 \leq i \leq r-2 \\
R_{i} R_{j} & =R_{j} R_{i}, \quad 1 \leq i, j \leq r-1, \quad|i-j| \geq 2 .
\end{aligned}
$$

Moreover, if $W$ is transitive, so is $R_{i} W$.

Proof. Straightforward case-checking.

In view of Proposition 2, we may define a group homomorphism

$$
R: \mathfrak{S}(r) \longrightarrow \operatorname{Aut~Walk}_{r}(\rho, \sigma)
$$

by mapping the Coxeter generator $(i i+1)$ to the operator $R_{i}$. We conclude that $\left(\operatorname{Walk}_{r}(\rho, \sigma), R\right)$ and $\left(\{2, \ldots, d\}^{r}, S\right)$ are permutation representations of $\mathfrak{S}(r)$, and that the diagram 
commutes for all $\pi \in \mathfrak{S}(r)$.

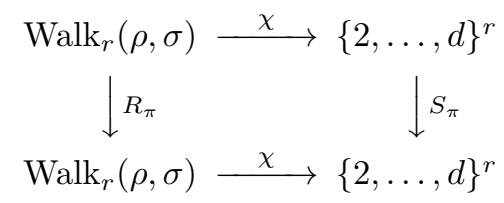

Proposition 3. The restriction of $\chi$ to any single orbit of $\left(\operatorname{Walk}_{r}(\rho, \sigma), R\right)$ is injective, with image the corresponding orbit of $\left(\{2, \ldots, d\}^{r}, S\right)$.

Proof. Since $\chi$ respects the action of $\mathfrak{S}(r)$, it sends each orbit of $\left(\operatorname{Walk}_{r}(\rho, \sigma), R\right)$ to an orbit of $\left(\{2, \ldots, d\}^{r}, S\right)$; the question is whether the image orbit is smaller than the source orbit, and we can answer it by looking at stabilizers.

Consider the element of the image orbit which is sorted, that is, the spectrum $\left(t_{1}, \ldots, t_{r}\right)$ such that $t_{1} \leq \cdots \leq t_{r}$. If the colour 2 appears $m_{2}$ times in the spectrum, the colour 3 appears $m_{3}$ times in the spectrum, and so on up to $m_{d}$, then the stabilizer of the spectrum is the Young subgroup

$$
\mathfrak{S}\left(m_{2}\right) \times \mathfrak{S}\left(m_{3}\right) \times \cdots \times \mathfrak{S}\left(m_{d}\right) \subseteq \mathfrak{S}(r) .
$$

This subgroup is generated by the transpositions $(i i+1)$ which exchange two adjacent copies of the same colour in the spectrum.

Now, consider a walk

$$
W=\left(\left(s_{1} t_{1}\right), \ldots,\left(s_{r} t_{r}\right)\right)
$$

with spectrum $\left(t_{1}, \ldots, t_{r}\right)$. By definition, we have $R_{i} W=W$ when $t_{i}=t_{i+1}$, so the stabilizer of $W$ contains the Young subgroup (2.4). Since $\chi$ is well-defined, the stabilizer of $W$ can be no bigger, so in fact it is exactly this Young subgroup. By the orbit-stabilizer theorem, the two orbits have the same size, so $\chi$ is a bijective correspondence between them, as needed.

As a further consequence, note that each orbit of $\left(\operatorname{Walk}_{r}(\rho, \sigma), R\right)$ contains a unique monotone walk, since each orbit of $\left(\{2, \ldots, d\}^{r}, S\right)$ contains a unique sorted spectrum.

2.2. Sorting colours. For any $c \in\{1, \ldots, d-1\}$, define $\vec{H}_{g}(\alpha, \beta ; c)$ to be the number of walks counted by $\vec{H}_{g}(\alpha, \beta)$ whose spectrum contains exactly $c$ distinct colours.

Proposition 4. For any $d \geq 1$ and $g \geq 0$, we have

$$
\sum_{c=1}^{d-1} 3^{c} \vec{H}_{g}\left(1^{d}, 1^{d} ; c\right) \leq \sum_{\alpha, \beta \vdash d} \vec{H}_{g}(\alpha, \beta) \leq \sum_{c=1}^{d-1} 4^{c} \vec{H}_{g}\left(1^{d}, 1^{d} ; c\right) .
$$

Proof. The monotone double Hurwitz number $\vec{H}_{g}(\alpha, \beta)$ counts monotone transitive walks

$$
\sigma=\rho\left(s_{1} t_{1}\right) \ldots\left(s_{r_{g}(\alpha, \beta)} t_{r_{g}(\alpha, \beta)}\right), \quad t_{1}<\cdots<t_{r_{g}(\alpha, \beta)}
$$

on $\mathfrak{S}(d)$ which begin at a permutation $\rho$ of cycle type $\alpha$, end at a permutation $\sigma$ of cycle type $\beta$, and take a total of $r_{g}(\alpha, \beta)=2 g-2+\ell(\alpha)+\ell(\beta)$ steps. Given such a walk, there is a unique strictly monotone walk 


$$
\rho=\operatorname{id}\left(s_{1}^{\rho} t_{1}^{\rho}\right) \ldots\left(s_{r_{0}(\alpha)}^{\rho} t_{r_{0}(\alpha)}^{\rho}\right), \quad t_{1}^{\rho}<\cdots<t_{r_{0}(\alpha)}^{\rho},
$$

from the identity permutation to $\rho$, and this walk is of length $r_{0}(\alpha)=d-\ell(\alpha)$. This fact, in different language, is due independently to Jucys [13, and Diaconis and Greene [3]; see [14] for a proof. Similarly, there is a unique strictly monotone walk

$$
\sigma=\operatorname{id}\left(s_{1}^{\sigma} t_{1}^{\sigma}\right) \ldots\left(s_{r_{0}(\beta)}^{\sigma} t_{r_{0}(\beta)}^{\sigma}\right), \quad t_{1}^{\sigma}<\cdots<t_{r_{0}(\beta)}^{\sigma},
$$

from the identity to $\sigma$, and this walk has length $r_{0}(\beta)=d-\ell(\beta)$. Thus the walk 2.5 gives rise to a transitive, identity based loop

$$
\mathrm{id}=\mathrm{id}\left(s_{1}^{\rho} t_{1}^{\rho}\right) \ldots\left(s_{r_{0}(\alpha)}^{\rho} t_{r_{0}(\alpha)}^{\rho}\right)\left(s_{1} t_{1}\right) \ldots\left(s_{r_{g}(\alpha, \beta)} t_{r_{g}(\alpha, \beta)}\right)\left(s_{r_{0}(\beta)}^{\sigma} t_{r_{0}(\beta)}^{\sigma}\right) \ldots\left(s_{1}^{\sigma} t_{1}^{\sigma}\right)
$$

of length

$$
r_{0}(\alpha)+r_{g}(\alpha, \beta)+r_{0}(\beta)=2 g-2+2 d=r_{g}\left(1^{d}, 1^{d}\right),
$$

which is thus a transitive loop of genus $g$. This loop, however, is not monotone; its spectrum

$$
\left(t_{1}^{\rho}, \ldots, t_{r_{0}(\alpha)}^{\rho}, t_{1}, \ldots, t_{r_{g}(\alpha, \beta)}, t_{r_{0}(\beta)}^{\sigma}, \ldots, t_{1}^{\sigma}\right)
$$

satisfies the inequalities

$$
\begin{aligned}
& t_{1}^{\rho}<\cdots<t_{r_{0}(\alpha)}^{\rho} \\
& t_{1} \leq \cdots \leq t_{r_{g}(\alpha, \beta)} \\
& t_{r_{0}(\beta)}^{\sigma}>\cdots>t_{1}^{\sigma} .
\end{aligned}
$$

Let $C^{\rho}=\left\{t_{1}^{\rho}, \ldots, t_{r_{0}(\alpha)}^{\rho}\right\}$ be the set of colours which appear in the first part of the spectrum of the transitive genus $g$ loop (2.6), and similarly let $C^{\sigma}=\left\{t_{1}^{\sigma}, \ldots, t_{r_{0}(\beta)}^{\sigma}\right\}$ be the set of colours which appear in the last part of the spectrum of this loop. Let us use the sorting action of $\mathfrak{S}\left(r_{g}\left(1^{d}, 1^{d}\right)\right)$ to rearrange the steps of 2.6 in weakly increasing order:

$$
\mathrm{id}=\mathrm{id}\left(s_{1}^{\prime} t_{1}^{\prime}\right) \ldots\left(s_{\left.r_{g}\left(1^{d}, 1^{d}\right)\right)}^{\prime} t_{r_{g}\left(1^{d}, 1^{d}\right)}^{\prime}\right), \quad t_{1}^{\prime} \leq \cdots \leq t_{\left(r_{g}\left(1^{d}, 1^{d}\right)\right)}^{\prime} .
$$

So far, this procedure is reversible - given the sorted loop (2.7) and the sets $C^{\rho}$ and $C^{\sigma}$, we can reconstruct the transitive loop $(2.6)$, and hence the original walk 2.5. Thus, to establish the stated inequalities, it suffices to show that, for every identity-based loop of length $2 g-2+2 d$ with $c$ colours in its spectrum, there are between $3^{c}$ and $4^{c}$ pairs of subsets $C^{\rho}, C^{\sigma}$ for which the reverse construction succeeds. Consider the possibilities for each of the $c$ colours in the spectrum:

(1) the colour appears in neither $C^{\rho}$ nor $C^{\sigma}$;

(2) the colour appears in $C^{\rho}$ but not $C^{\sigma}$;

(3) the colour does not appear in $C^{\rho}$ but appears in $C^{\sigma}$; or

(4) the colour appears in both $C^{\rho}$ and $C^{\sigma}$. 
Possibilities (1-3) are always fine for the reverse construction, whereas option (4) only works when the colour appears at least twice in the spectrum. Thus, there is an independent choice of at least 3 and at most 4 options for each of the $c$ colours, resulting in between $3^{c}$ and $4^{c}$ valid pairs $C^{\rho}, C^{\sigma}$.

Proposition 4 establishes the inequality (1.5), and thus completes the proof of Theorem 1 .

\section{REFERENCES}

1. P. Biane, Parking functions of types $A$ and B, Electron. J. Combin. 9 (2002), \#N7.

2. B. De Wit, G. 't Hooft, Nonconvergence of the $1 / N$ expansion for $S U(N)$ gauge fields on a lattice, Phys. Lett. B 69, 61-64.

3. P. Diaconis, C. Greene, Applications of Murphy's elements, Stanford University Technical Report 335 (1989).

4. N. Do, A. Dyer, D. Matthews, Topological recursion and the quantum curve for monotone Hurwitz numbers, arXiv:1408.3992.

5. N. Do, M. Karev, Monotone orbifold Hurwitz numbers, arXiv:1505.06503.

6. D. Eisenbud, N. Elkies, J. Harris, R. Speiser, On the Hurwitz scheme and its monodromy, Compositio Mathematica 77 (1991), 95-117.

7. I. P. Goulden, M. Guay-Paquet, J. Novak, Monotone Hurwitz numbers in genus zero, Canad. J. Math. 65 (2013), 1020-1042.

8. I. P. Goulden, M. Guay-Paquet, J. Novak, Polynomiality of monotone Hurwitz numbers in higher genera, Adv. Math. 238 (2013), 1-23.

9. I. P. Goulden, M. Guay-Paquet, J. Novak, Monotone Hurwitz numbers and the HCIZ integral, Ann. Math. Blaise Pascal 21 (2014), 71-99.

10. I. P. Goulden, D. M. Jackson, R. Vakil, Towards the geometry of double Hurwitz numbers, Adv. Math. 198 (2005), 43-92.

11. D. J. Gross, W. Taylor IV, Two-dimensional $Q C D$ is a string theory, Nucl. Phys. B 400 (1993), 181-208.

12. A. Hurwitz, Ueber Riemann'sche Flächen mit gegebenen Verzweigungspunkten, Math. Ann. 39 (1891), 1-60.

13. A. Jucys, Symmetric polynomials and the center of the symmetric group ring, Rep. Math. Phys. 5 (1974), 107-112.

14. S. Matsumoto, J. Novak, Jucys-Murphy elements and unitary matrix integrals, Internat. Math. Res. Not. 2 (2013), 362-397.

15. J. Novak, Jucys-Murphy elements and the Weingarten function, Banach Cent. Publ. 89 (2010), 231-235.

16. J. Novak, Lozenge tilings and Hurwitz numbers, J. Stat. Phys. 161 (2015), 509-517.

17. A. Okounkov, Toda equations for Hurwitz numbers, Math. Res. Lett. 7 (2000), 447-453.

18. A. Okounkov, R. Pandharipande, Gromov-Witten theory, Hurwitz theory, and completed cycles, Ann. Math. 163 (2006), 517-560.

19. A. Okounkov, R. Pandharipande, Gromov-Witten theory, Hurwitz theory, and matrix models, arXiv:0101147v2.

20. L. Pyber, Enumerating finite groups of given order, Ann. Math. 137 (1993), 203-220.

21. S. Samuel, $U(N)$-integrals, $1 / N$, and the De Wit-'t Hooft anomalies, J. Math. Phys. 21 (1980), 2695-2703.

22. R. P. Stanley, Parking functions and noncrossing partitions, Electron. J. Combin. 4 (1997), \#R20.

Department of Combinatorics \& Optimization, University of Waterloo, Canada

E-mail address: ipgoulden@uwaterloo.ca

LACIM, Université du Québec À Montréal, Canada

E-mail address: mathieu.guaypaquet@lacim.ca

Department of Mathematics, University of California, San Diego

E-mail address: jinovak@ucsd.edu 